\title{
Crítica a las genealogías reales e imaginarias
}

\section{Real and imaginary genealogies critique}

Mónica Humeres (monicahumeresr@gmail.com) Facultad de Ciencias Sociales, Universidad Alberto Hurtado (Santiago, Chile) ORCID: 0000-0002-9555-035X

\begin{abstract}
Currently the term genealogy is widespread in the social sciences as a methodology used to explain contemporary problems. However, genealogy, as a form of knowledge, is used in different ways. Contemporary philosophers such as Bernard Williams and Edward Craig have addressed different genealogical perspectives, characterizing the qualities and possibilities offered by each and identifying two types: what they call real genealogies and imaginary or fictional genealogies. In this article I argue that such a classification is problematic, because the term genealogy substantially refers to something that cannot be imaginary or fictional. The genealogist's way of knowing is based on recollecting pieces of real history that will allow him or her to look at the subject matter from a new perspective; therefore, to speak of imaginary or fictional genealogies is contradictory.
\end{abstract}

Key words: genealogy, history, epistemology, methodology, knowledge.

\section{Resumen}

Actualmente el término genealogía se encuentra bastante extendido en las ciencias sociales como una metodología empleada para explicar problemas contemporáneos. Con todo, la genealogía, en tanto forma de conocimiento, se emplea de maneras disímiles. Filósofos contemporáneos como Bernard Williams y Edward Craig han abordado distintas perspectivas genealógicas, caracterizando las cualidades y posibilidades que ofrece cada una e identificando así dos tipos: lo que denominan genealogías reales y genealogías imaginarias o ficcionales. En este artículo argumento que dicha clasificación es problemática pues, el término genealogía sustancialmente hace referencia a algo que no puede ser imaginario o ficcional. La forma de conocer del genealogista se basa en la recolección de trozos de historia real que permitirán mirar el objeto estudiado desde una nueva perspectiva, por lo tanto, hablar de genealogías imaginarias o ficcionales resulta contradictorio.

Palabras clave: genealogía, historia, epistemología, metodología, conocimiento.

\section{Introducción}

Dado que en ciencias sociales se ha ido popularizando cada vez más la genealogía como un método de investigación histórica -pero que difiere de la mera historicidad, predominando el interés por dar explicación a una cuestión del presente- sus usos e incluso su carácter ha ido variando de tal modo que llega a emplearse de modos contradictorios. Actualmente en el campo de la filosofía contemporánea se están empleando las genealogías imaginarias o ficcionales para dar explicación al devenir de ciertos 
objetos materiales o bienes epistémicos. Autores como Bernard Williams y Edward Craig equiparan las genealogías imaginarias o ficcionales a lo que llaman genealogías reales. Así, el argumento central de este artículo es que las genealogías imaginarias y las reales son desiguales en su esencia y responden a inquietudes de muy distinta índole, incluso antitéticas. Dado que las genealogías reales emergieron en contestación a un tipo de filosofía a-histórica, la tipificación de las genealogías imaginarias sugiere una separación profunda -y desde mi punto de vista- difícilmente reconciliable entre ambas genealogías. Para fundamentar mi tesis recurriré a una cita de Foucault ampliamente difundida, que versa sobre el carácter gris de las genealogías. Esta cita tiene su base en el trabajo de Nietzsche y en ella se encuentra el atributo medular de las genealogías reales, lo que permitirá caracterizar los aspectos fundamentales de la perspectiva real.

El cuerpo de este trabajo se compone principalmente de tres partes. (I) Comenzaré por esbozar el uso del método genealógico por los filósofos contemporáneos, principalmente de Bernard Williams y Edward Craig, distinguiendo entre genealogías reales e imaginarias, (II) para luego contrastar esta perspectiva con el trabajo de Nietzsche y Foucault, sin duda los autores más citados por los filósofos contemporáneos como precursores de la genealogía. Este trazado que me llevará a dirimir que los dos tipos de genealogías presentadas, imaginarias y reales, responden a intereses de investigación contrapuestos, por lo tanto, al compararlas o exponerlas como dos tipos de una misma genealogía, se arriesga trastocar el carácter de una u otra. (III) Con todo, hacia el final veremos que existen ciertos matices donde es posible, como bien hace Williams, intercambiar algunas de sus características. Con todo, el argumento general de este artículo consiste que las genealogías imaginarias y reales son dos metodologías intrínsecamente diferentes, por lo tanto, su intercambiabilidad o comparación no parece pertinente.

\section{Epistemología, genealogía y el estado de naturaleza}

Comúnmente cuando se emplea el método genealógico para conocer un bien epistemológico, se orienta a adquirir y mejorar ciertas creencias y su warranty [garantía]. Los estudios genealógicos en el campo de la epistemología generalmente están vinculados al Estado de Naturaleza [State of Nature], siendo también denominadas genealogías imaginarias o ficcionales. En este espectro encontramos a investigadores como Edward Craig y Bernard Williams, quienes sitúan sus perspectivas genealógicas en una relación de herencia con el naturalismo, aunque con grados diferentes de afinidad.

Por una parte, Craig establece claramente su relación con el Estado de Naturaleza situándose en el naturalismo, en tanto se piensa al hombre, "su comportamiento e instituciones, como hechos naturales que deben entenderse como el resultado -en términos causales generales- de otros hechos naturales" (Craig 2002:9). De este modo, el autor se pregunta principalmente por el conocimiento en su Estado de Naturaleza, buscando identificar las necesidades humanas que la emergencia de un concepto, como el de conocimiento, vino a satisfacer -también en sus formas más primitivas- así como por su devenir en el tiempo. Su preocupación epistémica es clara. Craig argumenta que los seres humanos necesitan creencias verdaderas acerca de su ambiente, creencias que pueden servir para guiar sus acciones a un resultado exitoso. Siendo así, los humanos requieren fuentes de información que los guíen a creer en esas verdades $y$, consecuentemente, el conocimiento vendría a satisfacer estas necesidades, evolucionando desde un concepto más primitivo de buen informante. En términos generales, Craig señala el conocimiento como una respuesta a la necesidad de evaluar y señalar las fuentes verídicas de información, aprobadas por una comunidad específica. 
En su libro Verdad y veracidad, Williams le dedica un apartado especial a explicar su uso del método genealógico. De esta manera, lo define como una narrativa que intenta explicar un fenómeno cultural mediante la descripción de la manera en que este devino, o que podría haber devenido, o que puede ser imaginado deviniendo. Así, distingue entre genealogías reales y ficticias, las que claramente, responden a necesidades o intereses de distinta índole. Las genealogías ficticias o imaginadas -según explica el autorbuscan explicar un concepto, valor o institución, mostrando las maneras en que estos podrían haber devenido de manera simplificada apuntando así a su Estado de Naturaleza. Williams diferencia su uso de este concepto de otros empleados en filosofía política, como por ejemplo es el caso de Robert Nozick en su trabajo Anarchy, state and utopia. A diferencia de Nozick, para Williams, el Estado de Naturaleza contiene una sociedad, un grupo de humanos que interactúan, cooperan. El punto clave estaría en que desde la perspectiva de Williams los humanos tienen ciertas necesidades básicas, y en respuesta a ellas habrían desarrollado el concepto, valor o institución investigada por las genealogías imaginarias. De esta manera Williams le otorga un papel preponderante a la cultura.

Ciertamente, la perspectiva genealógica de Williams es más crítica con el naturalismo que Craig. Williams reprocha que el naturalismo parte de la idea -muy general- de que los hombres son parte de la naturaleza, dejando bastante a la interpretación. El punto de inflexión para Williams es que el sistema ético sea parte de lo que llamamos naturaleza. En general, comenta que se entiende por naturaleza lo que tradicionalmente las ciencias naturales han definido como tal, e incluso ahí, quedan márgenes de ambigüedad donde no queda claro cuáles son las fronteras de lo que se entiende por naturaleza. Asimismo, Williams sostiene que el naturalismo se equivoca en la manera de interpretar el resto de la naturaleza. Primero, sostiene que "necesitamos asumir el hecho de que la cultura afecta casi toda la psicología humana" (Williams 2006:36). De esta forma, Williams hace referencia a la manera en la cual un instinto de una especie funciona en el entorno cultural depende de la forma de vida de aquella especie, esto es, la cultura y no únicamente la naturaleza en un sentido a-cultural.

\section{Genealogías reales e imaginarias}

Las genealogías reales, tienen una base consistente en la obra de Friedrich Nietzsche, trabajo que retomó Michel Foucault. Así como Williams, son innumerables los autores que los citan a estos dos filósofos como referentes del método genealógico. La perspectiva iniciada por Nietzsche, y continuada por Foucault, consiste en hacer filosofía desde una mirada histórica y práctica, sustentada en la búsqueda de documentos, a modo de dar cuenta del pasado de un concepto u objeto -en su sentido más amplio- que existe en el presente. Es en este sentido que a estas genealogías se les denomina Historia del presente. En otros términos, se trata de describir la trayectoria, con sus continuidades y rupturas, de prácticas, valores o conceptos cotidianos. Con todo, no se puede obviar el carácter crítico de los fundadores de esta perspectiva real. Tanto Foucault como Nietzsche se interesaron por evidenciar las relaciones de poder en el establecimiento de la verdad y sus efectos. En este sentido, la genealogía revela que la emergencia de las cosas, casi siempre, es el fruto de conflictos, accidentes, contingencias. Se profundizará en esta perspectiva en el apartado siguiente.

Las genealogías imaginarias, por lo general, están dirigidas a ayudar a explicar un concepto, valor o institución, mostrando las formas en que estos podrían haber devenido en ambientes simplificados. Desde la perspectiva de Williams, las genealogías imaginarias ofrecen tres elementos: (1) una explicación funcional inesperada, basada en motivaciones de los seres humanos en general, (2) una contribución a la comprensión simplificada, pues la explicación funcional establece una relación entre una razón compleja 
y una motivación más simple, en un vínculo racional y, (3) el relato ilumina las implicancias o trascendencia del concepto investigado.

Las genealogías imaginarias tienen un uso extendido -aunque no preponderante- en la filosofía contemporánea, respondiendo a cuestiones relevantes en la materia. No obstante, no todas las genealogías imaginarias son iguales ni envuelven un mismo tipo de explicación. Dentro de la diversidad de genealogías imaginarias, encontramos explicaciones de los siguientes tipos:

Tal como se mencionó antes, la explicación funcional es una de las más aceptadas por los genealogistas de ficción. Esto es, realizar una genealogía para establecer una función del concepto investigado en relación con las necesidades más básicas de los humanos, contribuyendo a destacar las características que tiene dicho concepto. Williams, subraya que esto no quiere decir que las genealogías funcionales indiquen una función real, pero ayudarían a ver una función donde antes no se había pensado. Otro tipo de explicación es el de la mano invisible, en la que el relato se configura dándose a entender que el concepto investigado es el resultado del diseño intencional de alguien. Este tipo de explicaciones son las que elabora Nozick en referencia al origen del Estado. Klemens Kappel prefiere un tipo de explicación que él llama práctica, estableciendo que la emergencia de un concepto u objeto surge como respuesta a una necesidad práctica. Lo define de la siguiente manera:

(1) Dado un conjunto de hechos $\mathrm{H}$, y un conjunto de objetivos o intereses I, tenemos una necesidad específica $\mathrm{N}$.

(2) E es lo que en realidad llena la necesidad N (Kappel 2010:81).

La explicación funcional se diferencia de la -llamada- mano invisible, en el énfasis en la intencionalidad de la segunda, como si fuese una sola racionalidad la que hace existir las cosas/conceptos. Kappel y Williams interpretan de modo distinto la explicación ofrecida por Craig sobre el conocimiento. Mientras Kappel interpreta el enfoque de Craig como una explicación práctica, Williams lo denomina explicación funcional. Conviene mantener esta distinción en consideración pues Kappel elabora ciertos matices en su explicación que difieren de, o podrían confundir, el trabajo realizado por Williams. El tipo de genealogía imaginaria realizada por Craig sobre el conocimiento, o por Williams sobre la veracidad, tienen por objetivo explicar conceptos y motivaciones que han acompañado a los seres humanos, si no es a lo largo de toda su vida en sociedad o comunidad, durante buena parte de su historia. Distinto es el objeto empleado por Kappel para ilustrar su perspectiva práctica, como veremos a continuación.

Kappel expresa que una cuenta genealógica del tipo imaginaria se podría usar para explicar, por ejemplo, por qué tenemos autos. A priori indica que la explicación apuntaría a señalar que los autos existen como respuesta a la necesidad humana de trasladarnos (función de traslado) entre puntos muy distantes. 0 bien, señala que "al menos, es muy poco probable que tuviésemos autos si nunca hubiésemos tenido la necesidad de transporte a la que ellos responden" (Kappel 2010:71), lo que vendría a ser la explicación práctica de dicha función.

Como se observa, en el ejemplo precedente el objeto de estudio -la explicación de la existencia de los automóviles- es de un tipo totalmente distinto a los desarrollados por Craig y Williams. Los automóviles son un objeto tecnológico de aparición reciente en términos históricos y no un concepto epistémico de larga data, por lo tanto, el tipo de explicación tiene un estatus diferente. Kappel emplea un artefacto que tiene una evidente utilidad, cuya invención es perfectamente identificable en la historia. Aun así, 
basándome en la amplia gama de investigaciones realizadas por historiadores de la tecnología tales como Thomas Hughes en The network of power o David Nye en Electrifying America y por académicos de los estudios de la Ciencia Tecnología y Sociedad (por ejemplo Bruno Latour en su Investigación sobre el sistema de transporte parisino), me es posible afirmar que incluso los objetos tecnológicos no son traídos a existencia por razones prácticas en la mayoría de los casos. Una amplia gama de investigaciones similares registra cómo la mayoría de los artefactos han sido producto de la conjunción de innumerables piezas de azar, de accidentes, de contingencias políticas y conflictos, lo que no quita que posteriormente dichos inventos se adapten a, o muchas veces creen, necesidades humanas.

Kappel, de todos modos, sustenta su manera de explicar el devenir de las cosas basado en la explicación práctica, argumentando que, aunque esta explicación no sea verdadera, hace sentido. Sin embargo, esto contradice precisamente lo que Nietzsche quiso evitar, una filosofía que, pudiendo hacer sentido del mundo mediante la historia, completara el vacío de conocimiento mediante la ficción. Esto se desarrollará en el siguiente apartado.

Por lo tanto, el argumento de este trabajo consiste en que las genealogías imaginarias y las reales poseen un estatus diferente, respondiendo a inquietudes de muy distinta índole, a veces opuestas. De este modo, mezclar ambas perspectivas como si fuesen comparables o instalarlas en un nivel analítico similar trastoca los propósitos sobre los cuales cada una se erige. Para fundamentar mi argumento volveré a quien por primera vez empleó el concepto de genealogía, Friedrich Nietzsche.

\section{El tono gris de una genealogía real: Nietzsche y Foucault}

Comúnmente se cita la escasa descripción que Foucault plasmó sobre su perspectiva genealógica. Esto es, la famosa frase que describe la genealogía como una tarea "gris, meticulosa y pacientemente documentada" (Foucault 1984:76). Incluso es reiterada por Williams. Foucault nunca despliega una explicación de lo que el calificativo gris quiere decir exactamente, sin embargo, aunque no lo reconoce de manera explícita, Foucault está parafraseando a Nietzsche. Mi afán de reparar en esa cita se justifica en la idea de que ésta nos entrega una pista fundamental, una clave analítica para comprender la genealogía nietzscheniana y foucaultiana y, para diferenciar de una vez, las genealogías denominadas reales e imaginarias. Por lo tanto, me interesa esclarecer ¿̇a qué se refería exactamente Nietzsche con el término gris?

Nietzsche compone su método genealógico en consideración y crítica del trabajo del filósofo judío-alemán Paul Rée, así como a los "genealogistas de la moral" ingleses (principalmente psicólogos) de su época. Nietzsche estaba interesado en los mismos asuntos morales que éstos, pero tuvo la intención de preguntarse lo mismo de un modo diferente. En relación al trabajo de sus pares contemporáneos, Nietzsche declara que está en contra de "esas hipótesis inglesas que se pierden en el vacío, en el azul del cielo; ya que está claro qué color ha de ser cien veces más importante que el azul para un genealogista de la moral: me refiero al gris, es decir, a lo que se basta en documentos, a lo realmente verificable, a lo que ha existido efectivamente, en pocas palabras, a toda esa escritura jeroglífica, larga y difícil de descifrar, referente al pasado de la moral humana" (Nietzsche 1994:40).

De esta manera, queda claro que para Nietzsche no existía tal cosa como una genealogía imaginaria. Precisamente estaba preocupado por señalar las verdades históricas, lo realmente existente, mediante una documentación detallada: eso era para él lo gris en una genealogía. De igual manera, Nietzsche estaba profundamente interesado en conciliar historia y filosofía, pero su preocupación por la historia no era por la mera historicidad, sino que la empleaba como una forma de iluminar el presente. Su inquietud no era 
acumular datos que probasen una hipótesis, sino que dar cuenta de una inversión radical de las cosas, reinvirtiendo los valores. Nietzsche critica categóricamente a sus pares, a quienes acusa de pensar de un modo "esencialmente ahistórico" (Nietzsche 1994:49). Según él, quienes le habían dedicado atención al estudio de la moral hasta ese entonces llegaban a elaborar juicios de utilidad sobre el concepto de 'bueno' y 'malo', estableciendo la superioridad del hombre por la capacidad de fabricar tales conceptos. Nietzsche advirtió que, a diferencia de lo que los genealogistas ingleses pensaron, el concepto de bueno no fue creado por la valoración que de la bondad hacían otros, sino por los mismos buenos, es decir, los nobles, los poderosos y privilegiados que se valoraron a sí mismos como buenos en oposición a todo lo vulgar, plebeyo. Por lo tanto, "la utilidad no les importaba en lo más mínimo" (Nietzsche 1994:49). Así, la perspectiva utilitarista le resultaba la más ajena e inapropiada.

Más de un siglo después, sin embargo, filósofos como Williams y Craig emplean el término genealogía para establecer la utilidad o funcionalidad de ciertos conceptos fundamentales de la humanidad. Esta perspectiva contemporánea me parece problemática si se le declara heredera de la obra de Nietzsche. Es más, me parece que las genealogías reales y las genealogías imaginarias responden a propósitos totalmente distintos y, al asemejarlas, se incurre en el riesgo de trastocar el sentido de ambas o establecer comparaciones poco justas. Este argumento cobra fuerza al observar la obra de Foucault.

Al retomar el trabajo de Nietzsche para construir su enfoque genealógico, Foucault se sustentó precisamente en la crítica a la utilidad. Foucault especificó que la genealogía "debe registrar la singularidad de los eventos fuera de cualquier finalidad monótona [...rechazando] los desarrollos metahistóricos de las significaciones ideales" (Foucault 1984:76). Continuando la herencia de Nietzsche, Foucault comentó que el análisis genealógico muestra que el concepto de libertad, por ejemplo, es una invención de las clases dominantes y no fundamental a la naturaleza humana. Así, el genealogista precisamente necesita la historia para desenmascarar las 'quimeras del origen'. A Foucault le interesa mantener la sucesión de eventos en su propia dispersión, identificando los accidentes, la emergencia en tanto irrupción de fuerzas, que le dan nacimiento a aquellas cosas que continúan existiendo y que tienen valor en la actualidad y sin reconciliación con el pasado. En esta perspectiva, la genealogía no busca la identidad de las cosas, sino revelar su disipación y discontinuidades.

Pareciera, por lo tanto, que la genealogía de Nietzsche y de Foucault poco tienen que ver con el Estado de Naturaleza, la ficción y/o las genealogías imaginarias de Williams y Craig. Menos aún con las explicaciones prácticas de Kappel.

Tal como se explicó en el primer apartado de este ensayo, el uso de la genealogía imaginaria que hacen los filósofos contemporáneos como Craig y Williams tiene como finalidad explicar y/o comprender la utilidad de que la humanidad haya desarrollado a cierto concepto, valor o institución. Mientras las genealogías reales, y específicamente las inspiradas por el análisis de Nietzsche y Foucault, pretender ser disruptivas, quebrantar nuestras concepciones, hacer que lo que damos por hecho ya no sea tan natural y contribuir a que re-evaluemos nuestros valores. Asimismo, este último tipo de genealogías comúnmente implican un análisis crítico de la modernidad, como una condición que no representa precisamente un progreso, sino que como una coerción en pro del gobierno de los cuerpos. Las genealogías imaginarias, por su parte, contienen una visión más positiva de la historia.

Pero entonces, ¿por qué Williams compara las genealogías imaginarias con las genealogías (que llaman) reales, señalándolas como dos metodologías equiparables? 


\section{Conciliaciones entre genealogías imaginarias y reales}

Williams tiene una mirada crítica tanto de las genealogías puramente imaginarias como de las puramente reales y es por este motivo que las considera complementarias. Advierte que una mirada únicamente funcional de la historia, como la que proveen las genealogías imaginarias, nos podría hacer incurrir en un error, distorsionando la comprensión de nuestra propia situación cultural, llevándonos a un reduccionismo. Es así como plantea la necesidad de incluir la mirada real de la historia. Sobre los aprendizajes que el uso de la perspectiva imaginaria le han dejado en su propia investigación sobre la veracidad, Williams comenta que "aunque el análisis pretende mostrar algunas conclusiones que pueden alcanzarse mediante estos argumentos abstractos, también nos ayuda a ver lo que no se puede obtener de esa manera. Podemos ofrecer una explicación de la veracidad y de su valor, creo en el modo filosófico particular de una genealogía ficticia, pero veremos que ese análisis es esencialmente incompleto, y que a partir de cierto punto, o, más bien, de varios puntos diferentes, tendremos que orientarnos a una genealogía real: a las contingencias culturales y a la historia. Podríamos expresarlo diciendo que la filosofía solo llega hasta cierto punto, que ofrece cierto tipo de explicaciones pero no otras" (Williams 2006:46).

Así, es evidente que Williams, como Nietzsche y Foucault, también está interesado en que la filosofía se involucre en la historia. Williams argumenta que, en esta tarea, la filosofía no puede pretender ser demasiado pura. En su investigación sobre la veracidad, manifiesta que existen tres razones por las que debe recurrir a la historia real. La primera es que la genealogía imaginaria tiene un límite analítico: "la genealogía imaginaria revela un vacío en las motivaciones de la veracidad, un vacío que puede llenarse con uno o más valores o actitudes diferentes, y qué valores y actitudes cumplen esa función en un momento dado y en una sociedad determinada es una cuestión de hecho. En este sentido, la cultura y la historia ponen algo en el análisis abstracto, ficticio" (Williams 2006:48).

En segundo lugar, aunque en la misma línea, Williams explica que los desarrollos culturales ensanchan los horizontes de la genealogía imaginaria. Finalmente, indica que el proceso histórico, aunque requiere de comprensión filosófica, es autónomo, se trata de un devenir surgido de hechos completamente contingentes que deben ser considerados.

A la luz de estas declaraciones, se puede concluir que Williams se sitúa entre las genealogías reales e imaginarias que, a su modo de pensar, son complementarias. Con todo, Williams deja ver que existe una fricción entre estas dos perspectivas genealógicas. Comenta que las genealogías reales contienen una complicación intrínseca: cuando éstas están relacionadas con la vida ética y los valores -complejos depósitos de diferentes tradiciones y fuerzas sociales- el genealogista se encontrará con que los cambios históricos que trajeron a existencia el objeto estudiado no están necesariamente relacionados a este, sino que son el producto accidental de otras trayectorias. Williams enfatiza que esta característica de contingencia tensiona una demanda fundamental de las ideas éticas: el reconocimiento de su autoridad. Asimismo, el sistema ético demanda transparencia. Para el autor, esta combinación, de autoridad y transparencia: "motivan una reflexión sobre sí mismos de una manera que pone de manifiesto su contingencia. Todo esto significa que podría haber algo impertinente, irrespetuoso o crítico en las explicaciones genealógicas" (Williams 2006:32).

Así, para Williams, una genealogía real ofrece una dificultad, pero también una ventaja sobre las genealogías puramente imaginarias: gracias a que se remite a una historia real, se adquiere un sentido más agudo de lo que está en juego con el desarrollo del punto de vista investigado. Con todo, a Williams 
le satisface combinar ambas perspectivas, con la salvaguarda de mantener "en su sitio los hechos históricos y la abstracción funcionalista" (Williams 2006:45).

Cabe mencionar que existen otros académicos que han intentado conciliar las distintas perspectivas genealógicas. Entre ellos, se puede mencionar a Miranda Fricker (Rational authority and social power), quien analiza ambos tipos de genealogías argumentando que el estudio genealógico puede ser diferenciado claramente desde una perspectiva histórica y otra filosófica. La autora indica que nos podemos concentrar en la simple distinción entre una búsqueda de explicación por parte de los historiadores ¿cómo ha ocurrido? y la búsqueda de la comprensión ¿cómo es posible? por parte de los filósofos. Sin embargo, la motivación elemental de la perspectiva genealógica de Nietzsche y Foucault precisamente consistía en no separar la historia de la filosofía y en cambio mantener una disciplina al servicio de la otra. Por lo tanto, la escisión que hace Fricker tampoco parece compatible con la definición que los genealogistas reales hicieron de sus trabajos.

\section{Conclusión}

En la filosofía contemporánea la genealogía imaginaria está marcada por su vínculo -más o menos presente- con el naturalismo y la idea de Estado de Naturaleza. Dentro de los aportes significativos de las genealogías imaginarias, se destaca su capacidad de contribuir a la comprensión del objeto investigado, estableciéndose una relación funcional entre una motivación simple de los humanos y una razón compleja. Asimismo, estas genealogías están conformadas por una narración que ilumina las implicancias o trascendencias del concepto investigado.

Por otra parte, las genealogías reales arraigadas en el trabajo de Nietzsche y continuado por Foucault, tienen como esencia el carácter gris: los hechos basados en documentos, lo realmente existente y verificable. Esta mirada pretende conciliar historia y filosofía, para dar cuenta de una inversión radical de las cosas, lejos de la utilidad o funcionalidad y de las significaciones ideales y para desenmascarar las 'quimeras del origen'. De esta manera, no se pretende dar cuenta de la unidad, sino de la dispersión de las cosas, como una provocación para repensar el presente.

Pareciera, por lo tanto, que la genealogía de Nietzsche y de Foucault poco tienen que ver con el Estado de Naturaleza, la ficción y/o las genealogías imaginarias de Williams y Craig. Más aún, las genealogías reales y las genealogías imaginarias responden a propósitos distintos y, por ello, su igualación (en tanto se les piensa como dos tipos de una misma cosa) arriesga alterar el sentido de una u otra.

En definitiva, aunque la combinación de la perspectiva imaginaria y real puede funcionar para algunos genealogistas contemporáneos, pareciera difícil que, al poner la genealogía real al servicio de la imaginaria, no se pierda el propósito de las genealogías: mantener unidas la filosofía y la historia, como dos prácticas inseparables para conocer el mundo. Asimismo, la sola clasificación de ambas genealogías como la subdivisión de una misma cosa, contiene en sí misma una fricción del carácter de ambas perspectivas. De esta manera, pareciera poco conveniente -incluso poco justa- la comparación o intercambiabilidad entre genealogía imaginaria y real. 


\section{Bibliografía}

Craig, E. 2002. Knowledge and the state of nature. An essay in conceptual synthesis. Oxford: Clarendon Press. https://doi.org/10.1093/0198238797.001.0001

Foucault, M. 1984. Nietzsche, genealogy, history, pp. 76-100. En: P. Rabinow. The Foucault reader. New York: Pantheon Books.

Kappel, K. 2010. On saying that someone knows: themes from Craig. En: A. Haddock et.al. Social epistemology. Oxford: Oxford University Press.

https://doi.org/10.1093/acprof:oso/9780199577477.001.0001

Nietzsche, F. 1994. Genealogía de la moral. Madrid: M.E Editores.

Williams, B. 2006. Verdad y veracidad. Barcelona: Tusquets Editores.

Recibido el 22 May 2018

Aceptado el 18 Sep 2018 